Collection SFN 11 (2010) 7-15

(C) Owned by the authors, published by EDP Sciences, 2010

DOI: $10.1051 / \mathrm{sfn} / 201011002$

\title{
Remarques sur la notion de cohérence en diffusion de neutrons aux petits angles
}

\author{
J. Teixeira
}

Laboratoire Léon Brillouin (CEA/CNRS) CEA Saclay 91191, Gif-sur-Yvette Cedex, France

\begin{abstract}
Résumé. La diffusion de neutrons aux petits angles (DNPA) est devenue une technique très courante, appliquée dans pratiquement tous les domaines de la physique de la matière condensée, de la chimie, de la biologie ou des sciences des matériaux. Les propriétés inhérentes à toute la diffusion neutronique (pouvoir de pénétration de matériaux denses, discrimination isotopique, diffusion magnétique) jouent un rôle capital dans l'universalité des applications de la DPNA. Cela ne va pas sans certains inconvénients, dont le principal est l'inexistence d'une théorie suffisamment générale pour être applicable à toutes les situations.

En conséquence, et malgré l'existence d'excellents ouvrages de référence, la littérature sur la DPNA concerne le plus souvent un seul secteur du vaste domaine des applications, développant ainsi des concepts spécifiques ou peu généralisables. Il faut prendre cette situation comme une richesse même si elle constitue une barrière certaine, par exemple, à la constitution de bibliothèques et de procédures de traitement de données générales dans les Très Grandes Installations.

Le propos de ce court article n'est certainement pas d'aborder ce problème, d'ailleurs probablement sans solution, mais de discuter quelques principes parfois oubliés, non sans mauvaises conséquences.
\end{abstract}

\section{COHÉRENCE}

Le mot "cohérence" est très souvent utilisé en physique et dans la DNPA avec des significations relativement différentes. Le propos de la quasi totalité des expériences de DNPA est de mesurer la section efficace cohérente d'un échantillon et de l'associer aux éventuelles cohérences spatiales présentes. Celles ci peuvent avoir des origines très diverses, depuis la présence d'une deuxième phase au sein d'une phase homogène, jusqu'à de simples fluctuations statistiques pas complètement aléatoires. Dans ce contexte, la cohérence est celle existante dans l'échantillon indépendamment de la sonde utilisée pour la détecter. La DNPA peut détecter les cohérences présentes dans l'échantillon via une onde quasi plane qui garde la même phase sur un domaine suffisamment étendu pour inclure la cohérence spatiale présente dans l'échantillon. Cela dépendra aussi d'une relation entre la cohérence de l'échantillon et la fonction de couplage avec le rayonnement. Plus exactement, avec différentes techniques on n'observera pas forcément les mêmes domaines de cohérence. Ainsi, par exemple, une séparation de phase entre isotopes (cas du polystyrène deutéré et hydrogéné) qui se traduit par des domaines plus riches en un isotope sera visible par DNPA et pas aux rayons X (du moins, dans la mesure où la différence de densité entre domaines n'est pas suffisamment importante pour être détectée).

Au contraire de ce qui passe avec le rayonnement laser, le faisceau de neutrons n'est pas cohérent. On ne saurait même pas parler d'un rayonnement. Un calcul élémentaire montre que, dans une situation typique où un flux monochromatique de $4 \AA$ de $10^{6}$ neutrons. $\mathrm{cm}^{-2} \cdot \mathrm{s}^{-1}$ est envoyé sur un échantillon, la densité de neutrons dans le faisceau incident n'est que de 10 particules par $\mathrm{cm}^{3}$. Chacun des neutrons est donc une particule classique et isolée dans le faisceau incident. Pourtant, au sein de l'échantillon, la densité de la matière et la portée des interactions imposent de traiter chaque neutron comme une onde dont les propriétés sont définies par le moment et par la collimation. En DNPA,

This is an Open Access article distributed under the terms of the Creative Commons Attribution-Noncommercial License 3.0, which permits unrestricted use, distribution, and reproduction in any noncommercial medium, provided the original work is properly cited. 
seule la cohérence transversale est vraiment importante. Un calcul de l'incertitude $\Delta k$ résultante de diaphragmes de $1 \mathrm{~cm}$ de diamètre sur une longueur de quelques mètres montre que l'extension de l'onde plane est de l'ordre de $1 \mu \mathrm{m}$, donc capable de déceler les cohérences de taille inférieure présentes dans l'échantillon.

Chaque neutron constitue donc une onde plane qui sonde une fraction substantielle de l'échantillon. Se couplant avec un grand nombre de noyaux, c'est l'interférence des très nombreuses ondes sphériques qui fournit l'information recherchée sur les corrélations spatiales. Bien sûr, le neutron incident ne produira qu'un neutron émergent dont le moment est imprévisible mais avec la probabilité imposée par le facteur de structure, $S(Q)$, de l'échantillon. C'est l'accumulation statistique d'un nombre suffisamment élevé de neutrons diffusés qui fournira une information fiable sur $S(Q)$. Pour terminer, on peut aussi noter que l'onde plane associée à un même neutron a une polarisation aléatoire mais qui se couple à la fois de manière parallèle et antiparallèle avec les spins nucléaires des atomes de l'échantillon. La séparation de la diffusion "cohérente" et "incohérente" (au sens de la technique neutronique) se fait, a posteriori, de manière statistique, avec les règles de la Mécanique Quantique.

La pratique de la diffusion avec des neutrons et/ou de noyaux polarisés permettrait des études plus détaillées mais est peu ou pas du tout pratiquée à cause de la faible intensité des faisceaux de neutrons et de l'accessibilité restreinte des installations.

\section{GÉNÉRALITÉS}

Le neutron dit thermique est une sonde de la matière parmi les plus intéressantes et riches de possibilités. En fait, le neutron est une particule neutre et qui n'interagit donc pas avec les champs électriques, dont celui dû aux électrons. De ce fait, la pénétration dans la matière est très facile même avec des énergies cinétiques très faibles, souvent inférieures à celle de l'agitation thermique des systèmes étudiés. Les informations sur la structure et la dynamique des échantillons étudiés par diffusion de neutrons proviennent donc exclusivement des forces nucléaires entre les neutrons et les noyaux des atomes.

Une autre interaction importante est celle du neutron avec l'induction magnétique du milieu traversé, dont celle qui résulte d'électrons dépareillés dans les matériaux magnétiques. Cette introduction n'abordera pas l'interaction magnétique, malgré son énorme intérêt.

Pour saisir globalement la nature des interactions neutrons - matière, il est utile de rappeler quelques unes des propriétés du neutron :

$$
\begin{aligned}
& \text { Masse }=1.675 \times 10^{-24} \mathrm{~g} \\
& \text { Charge }=0 \\
& \text { Demi-vie: } 886.8 \mathrm{~s}(14.8 \text { minutes }) \\
& \text { Spin }=1 / 2 \\
& \text { Moment dipolaire: }-1.913 \mu_{\mathrm{B}} .
\end{aligned}
$$

Les expériences de diffusion de neutrons utilisent exclusivement des neutrons "thermiques", ce qui veut dire que leur vitesse a été préalablement diminuée ("modérée") par interaction inélastique avec un milieu "thermalisé" à des températures adéquates: $20 \mathrm{~K}$ (thermalisation à l'hydrogène liquide, neutrons "froids"), $300 \mathrm{~K}$ (neutrons "thermiques"), $1200^{\circ} \mathrm{C}$ (neutrons "chauds"). Dans ces domaines de basse énergie, il n'y a presque jamais lieu de tenir compte de termes de résonance ou des niveaux d'énergie des noyaux dans l'interaction neutron -noyau.

En tenant compte de la masse $m$ du neutron et de la température $T$, on peut facilement déduire la vitesse $v$ des neutrons. La relation de de Broglie qui relie le moment $m v$ d'une particule à celui, $k$, d'une onde plane, donc à une longueur d'onde $(m v=h k / 2 \pi=h \lambda)$, permet d'écrire les relations suivantes, où $E$ est l'énergie, $h$ la constante de Planck, $k_{B}$ la constante de Boltzmann et $\lambda$ la longueur d'onde. 


$$
\begin{aligned}
& E=\left(k_{B} T\right)=\frac{1}{2} m v^{2}=\left(\frac{h^{2}}{2 m}\right) \frac{1}{\lambda^{2}} \\
& \lambda=6.283 \frac{1}{k}=3.956 \frac{1}{v}=9.045 \frac{1}{\sqrt{E}} \\
& E=5.227 v^{2}=81.81 \frac{1}{\lambda^{2}}=2.072 k^{2} .
\end{aligned}
$$

La relation énergie - température figure entre parenthèses parce que, physiquement elle devrait être égale à $3 k_{B} T / 2$, mais la "tradition" oblige à l'écrire de cette manière. Cela est sans conséquences, puisque de toute manière les neutrons disponibles ont un spectre très large plus ou moins maxwellien et, en pratique, le paramètre intéressant est la longueur d'onde, souvent imposée par monochromatisation du faisceau incident, où déduite de la vitesse.

Il est important de constater les domaines numériques (voir le Tableau ci-dessous) des paramètres qui résultent des équations ci-dessus, pour les neutrons thermiques. La "fenêtre" de longueur d'onde s'étend de 0.5 à $20 \AA$, des valeurs comparables aux distances inter-atomiques; la fenêtre d'énergie est, par construction, comparable aux énergies cinétiques. Ces deux caractéristiques rendent la diffusion de neutrons une technique de choix pour l'étude de la structure de la matière à l'échelle atomique, ainsi que pour l'étude des mouvements atomiques et moléculaires (diffusions, rotations, vibrations, phonons, etc.).

\begin{tabular}{lcccc}
\hline$\lambda(\AA)$ & $\mathrm{v}(\mathrm{km} / \mathrm{s})$ & $\mathrm{E}(\mathrm{meV})$ & $\mathrm{E}(\mathrm{K})$ & $1 \AA / \mathrm{v}(\mathrm{ps})$ \\
\hline 1 & 3.96 & 81.81 & 949 & 0.025 \\
4 & 0.99 & 5.11 & 59.3 & 0.101 \\
10 & 0.40 & 0.818 & 9.49 & 0.252 \\
\hline
\end{tabular}

Rappelons que, dans le cas de la diffusion de la lumière visible, la longueur d'onde est de l'ordre du $\mu \mathrm{m}$ et que les rayons $\mathrm{X}$ ont des énergies très supérieures à $\mathrm{k}_{\mathrm{B}} \mathrm{T}$. Peut-être plus important est le fait que l'énergie varie avec le carré de la longueur d'onde offrant une plage d'étude en énergie très large. Dans le cas des ondes électromagnétiques, l'énergie dépend linéairement de la longueur d'onde.

Malgré donc de solides atouts, la diffusion de neutrons est le plus souvent une technique “complémentaire". Ceci résulte de la faible intensité des sources, des processus de thermalisation et de monochromatisation, des difficultés de focalisation des faisceaux. Les ordres de grandeur des flux moyens disponibles dans une installation moderne sont les suivants:

Flux au niveau du réflecteur du réacteur: $10^{15} \mathrm{n} \cdot \mathrm{cm}^{2} \cdot \mathrm{s}^{-1}$

Flux polychromatique après thermalisation: $10^{9} \mathrm{n} \cdot \mathrm{cm}^{2} \cdot \mathrm{s}^{-1}$

Flux monochromatique au niveau d'un échantillon: $10^{5}$ à $10^{7} \mathrm{n} \cdot \mathrm{cm}^{2} \cdot \mathrm{s}^{-1}$

Ces valeurs sont plusieurs ordres de grandeur inférieures à celles disponibles dans les installations courantes de diffusion de la lumière ou de rayons $X$, sans parler des grandes installations actuelles de rayonnement synchrotron. La conséquence logique est que la diffusion neutronique est réservée à des cas qui justifient absolument son utilisation, ce qui, pourtant, ne devrait pas limiter les demandes de temps de faisceau et la bonne apprentissage de la technique !

Si l'on part du flux disponible au niveau d'un échantillon, évoqué ci-dessus, on se rend compte que, pour un échantillon typique contenant environ $10^{22}$ atomes, il faudrait attendre des millions d'années avant que chaque site ait interagi avec un neutron. En réalité, la distance entre les neutrons dans le faisceau incident est très grande (de l'ordre $\mathrm{du} \mathrm{cm}$ ) et chacun peut être pris comme une particule classique dont on connaît et la position et le moment. Mais, dans l'échantillon, la distance typique entre atomes étant, comme nous l'avons vu, comparable à la longueur d'onde, on se trouve dans la situation où on connaît le moment du neutron (module de la vitesse imposée par la monochromatisation, direction 
imposée par la collimation) mais on ne connaît pas la position. Idéalement, chaque neutron est une onde plane d'extension infinie qui interagit avec tous les noyaux de l'échantillon. En pratique, l'extension est finie et imposée par la collimation (cohérence transversale) ce qui définit une "aire de cohérence" à l'intérieur de laquelle les déphasages dus aux interactions avec chaque atome peuvent être interprétés en termes de distances inter-atomiques. Pour les appareillages courants, cette aire de cohérence est de l'ordre du $\mu \mathrm{m}^{2}$. Notons que, néanmoins, le neutron est presque toujours détecté en tant que particule. L'interférence constructive "régénère" le neutron avec une loi de probabilité qui dépend des interactions et de la structure de l'échantillon. Une expérience visant la détermination de la structure doit se faire de manière à que l'aire de cohérence soit plus grande que les distances en jeu pour la définition de la structure à une échelle donnée.

Remarque: Ceci n'a strictement rien à voir avec la "résolution” expérimentale. Les faibles intensités des flux disponibles amènent à utiliser des neutrons dont la longueur d'onde est à l'intérieur d'une bande dont la largeur atteint parfois $10 \%$ de la longueur d'onde nominale, ce qui amène à une certaine résolution structurale. Mais, dans l'interaction neutron - matière, chaque neutron doit être pris individuellement.

La théorie de la diffusion des neutrons par les noyaux atomiques est assez simple du fait que ces derniers peuvent être considérés comme ponctuels $\left(10^{-17} \mathrm{~cm}\right)$. Le potentiel d'interaction est donc sphérique. La valeur typique de la section efficace de diffusion est de $10^{-24} \mathrm{~cm}^{2}=1 \mathrm{barn}$.

Les détails de la théorie se trouvent dans plusieurs livres et articles de synthèse. On ne rappellera ici que le processus de l'interaction, idéalisé pour un atome isolé. L'onde plane incidente interagit avec le noyau et génère une onde sphérique d'amplitude $b$ (la longueur de diffusion cohérente) :

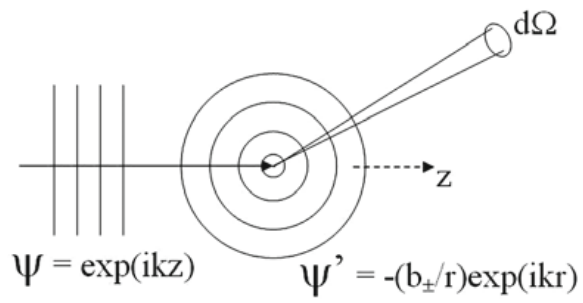

La section efficace cohérente est donnée par $\sigma_{c o h}=4 \pi b^{2}$.

Notons que cette représentation est schématique dans la mesure où, en pratique, la valeur de $b$ correspond à un atome "lié", c'est-à-dire, parmi les autres atomes de la matière.

Ce sont les interférences entre les ondes sphériques issues de plusieurs atomes qui, d'une manière bien connue, vont générer des interférences constructives dans certaines directions de l'espace.

Dans le schéma ci-dessus best affecté d'un indice \pm . Comme nous avons vu au début, le neutron a un spin $1 / 2$. Chaque noyau a un spin $I$. Dans l'interaction de l'onde plane avec chaque noyau le couplage peut se faire avec les spins parallèle (spin résultant $I+1 / 2$ ) ou anti-parallèle (spin résultant $I-1 / 2$ ). Dans chaque cas, l'interaction sera différente et il y a lieu, pour chaque noyau (chaque isotope) de définir deux valeurs de $b: b_{+}$(spins parallèles) et $b_{-}$(spins anti-parallèles), les seules exceptions étant les noyaux de spin nul. Certaines expériences utilisent des faisceaux "polarisés" où tous les spins des neutrons incidents ont la même direction suite à l'interaction avec un milieu polarisant. Dans d'autres cas, les spins nucléaires peuvent eux aussi être polarisés. La considération de ces cas dépasse le caractère élémentaire de cette introduction. Notons que, dans le cas le plus fréquent, ni les spins des neutrons incidents ni ceux de l'échantillon ne sont orientés. C'est donc "le même" neutron (onde plane, comme nous l'avons vu) qui, de manière aléatoire se couple avec chaque noyau, la statistique obéissant, bien sûr, aux règles de la mécanique quantique.

En pratique, on définit pour chaque isotope une valeur moyenne des longueurs de diffusion cohérente, moyenne pondérée de $b_{+}$et de $b_{-}$. C'est la valeur de la "longueur de diffusion cohérente" que l'on trouve sur les Tables. Pour les éléments naturels, on calcule aussi un $b$ moyen pondéré par 
la concentration isotopique. Les moyennes des écarts à ces valeurs définissent une "section efficace incohérente". La section efficace de diffusion d'un noyau est donc la somme d'un terme cohérent et d'un terme incohérent.

A titre d'exemple, le tableau suivant indique les valeurs des différentes valeurs évoquées pour l'hydrogène et pour le deutérium.

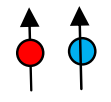

n I

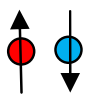

n I

\begin{tabular}{|l|c|c|}
\hline Spin de l'ensemble noyau-neutron & $\mathrm{I}+1 / 2$ & $|\mathrm{I}-1 / 2|$ \\
\hline Nombre d'états & $f_{+}=\frac{I+1}{2 I+1}$ & $f_{-}=\frac{I}{2 I+1}$ \\
\hline Longueur de diffusion & $\mathrm{b}_{+}$ & $\mathrm{b}_{-}$ \\
\hline
\end{tabular}

$$
\begin{gathered}
\bar{b}=f_{+} b_{+}+f_{-} b_{-} \\
\overline{b^{2}}=f_{+} b_{+}^{2}+f_{-} b_{-}^{2} \\
\sigma_{c o h}=4 \pi(\bar{b})^{2} \\
\sigma_{i n c}=4 \pi\left[\overline{b^{2}}-(\bar{b})^{2}\right] \\
\sigma_{s}=\sigma_{c o h}+\sigma_{i n c} \\
\left(1 \text { barn }=10^{-24} \mathrm{~cm}^{-2}\right)
\end{gathered}
$$

\begin{tabular}{|l|c|c|}
\hline & $\mathrm{H}$ & $\mathrm{D}$ \\
\hline $\mathrm{I}$ & $1 / 2$ & 1 \\
\hline $\mathrm{I}+1 / 2$ & 1 & $3 / 2$ \\
\hline $\mathrm{I}-1 / 2$ & 0 & $1 / 2$ \\
\hline $\mathrm{b}_{+}\left(10^{-12} \mathrm{~cm}\right)$ & 1.085 & 0.953 \\
\hline $\mathrm{b}_{-}\left(10^{-12} \mathrm{~cm}\right)$ & -4.750 & 0.098 \\
\hline $\mathrm{f}_{+}$ & $3 / 4$ & $2 / 3$ \\
\hline $\mathrm{f}_{-}$ & $1 / 4$ & $1 / 3$ \\
\hline $\bar{b}\left(10^{-12} \mathrm{~cm}\right)$ & -0.374 & 0.668 \\
\hline$\overline{b^{2}}($ barn $)$ & 6.523 & 0.609 \\
\hline$\sigma_{\text {coh }}($ barn $)$ & 1.758 & 5.607 \\
\hline$\sigma_{\text {inc }}($ barn $)$ & 79.91 & 2.04 \\
\hline$\sigma_{\mathrm{s}}($ barn $)$ & 81.67 & 7.65 \\
\hline
\end{tabular}

Le cas de l'hydrogène est unique en ce qui concerne la section efficace incohérente. Le plus souvent les études structurales de matériaux hydrogénés sont difficiles, entachées d'erreurs inhérentes, voire impossibles. Mais, elle ouvre la possibilité de l'étude très précise de dynamiques individuelles de matériaux hydrogénés, puisque toute contribution cohérente y est négligeable.

Notons enfin que la substitution isotopique, se traduisant éventuellement par des diffusions cohérentes diverses, ouvre des possibilités de "marquage" d'atomes, de molécules ou de fragments d'agrégats d'une grande richesse potentielle.

\section{L'APPROXIMATION STATIQUE}

La DNPA, comme toutes les expériences de diffraction, prétend mesurer le facteur de structure $S(Q)$. En fait, l'intensité diffusée, $S(Q, \omega)$, est la transformée de Fourier temporelle de la fonction intermédiaire $I(Q, t)$ qui s'écrit :

$$
I(\vec{Q}, t)=\sum_{i, j} b_{i} b_{j}\left\langle\exp \left[i \vec{Q} \cdot \vec{r}_{i}(0)\right] \exp \left[-i \vec{Q} \cdot \vec{r}_{i}(t)\right]\right\rangle+\sum_{i}\left(b_{i}^{i n c}\right)^{2}\left\langle\exp \left[i \vec{Q} \cdot \vec{r}_{i}(0)\right] \exp \left[-i \vec{Q} \cdot \vec{r}_{i}(t)\right]\right\rangle .
$$

Dans cette expression, $b_{i}$ est la longueur de diffusion cohérente de l'atome $i$ et le deuxième terme représente la contribution incohérente. Les parenthèses $\langle\ldots\rangle$ représentent des moyennes sur l'ensemble. $\vec{Q}$ est l'échange de moment et $\vec{r}_{i}(t)$ est la position de l'atome $i$ à l'instant $t$. 
L'approximation statique consiste à supposer que le temps d'interaction entre l'onde plane et l'ensemble des noyaux (à ne pas confondre avec le temps d'interaction avec chaque noyau) est suffisamment court pour que l'on puisse négliger les mouvements des atomes. Cela correspond à éliminer la dépendance en $t$ de l'équation précédente, c'est à dire à considérer que l'expérience correspond à une mesure "instantanée" de la structure de l'échantillon. On aboutit alors à l'expression :

$$
S(Q)=\sum_{i, j} b_{i} b_{j} \exp \left[i \vec{Q} \cdot\left(\vec{r}_{i}-\vec{r}_{j}\right)\right]+\sum_{t}\left(b_{i}^{i n c}\right)^{2}
$$

avec

$$
S^{c o h}(Q)=\sum_{i, j} b_{i} b_{j} \exp \left[i \vec{Q} \cdot\left(\vec{r}_{i}-\vec{r}_{j}\right)\right]^{2}
$$

où la dépendance temporelle ne figure plus et la contribution due à la diffusion incohérente se réduit à une constante additive.

En réalité, l'onde plane traverse l'échantillon dans une direction presque perpendiculaire au vecteur $\vec{Q}$. Les interactions ont donc lieu sur un intervalle de temps qui dépend de la vitesse et, donc, de la longueur d'onde. Ainsi, pour un faisceau incident monochromatique de $4 \AA$, cette vitesse est de l'ordre de 1000 m/s, soit $10 \AA ̊$ Àps. L'étude de structures disons de l'ordre de $100 \AA$ A par DNPA suppose implicitement qu'elles n'évoluent sur des temps de l'ordre de $10 \mathrm{ps,}$, ce qui est raisonnable si l'on tient compte des temps caractéristiques de diffusion de telles structures mais n'est pas forcément vrai, raison pour laquelle l'hypothèse de l'approximation statique ne saurait être complètement ignorée.

Dans une expérience de DNPA, un détecteur idéal intègre toute l'intensité diffusée dans une direction faisant un angle $\theta$ avec la direction du faisceau incident quelle que soit l'échange d'énergie, $\Delta \omega$, ce qui correspond à intégrer l'intensité diffusée le long d'une ligne de dispersion dans le plan $(Q, \omega)$. Cette intégration est le plus souvent identifiée à une intégration sur tous les échanges d'énergie qui auraient eu lieu à $Q$ constant. Surtout en DNPA, la contribution élastique $(\omega=0)$ est dominante, ce qui revient à dire que l'on fait les approximations :

$$
S(Q)^{\text {mesuré }} \approx S(Q) \approx S(Q, \omega=0)
$$

\section{FACTEURS DE FORME ET FACTEURS DE STRUCTURE}

La diffusion de neutrons, comme toute technique de diffusion, analyse les propriétés d'un échantillon dans un espace réciproque, l'espace où le transfert de moment est représenté par la variable. L'intensité diffusée est proportionnelle aux périodicités de la structure, elle aussi une notion définie dans l'espace réciproque, qui est la transformée de Fourier des corrélations spatiales présentes dans l'échantillon. Une erreur fréquente consiste à prendre l'intensité diffusée $I(Q)$ comme s'il s'agissait de la transformée de Fourier d'un cliché de microscopie, quand en fait il s'agit (en première approximation) de la transformée de Fourier des corrélations du cliché. Cette erreur est courante en DNPA, probablement parce que, dans un cas particulier, $I(Q)$ est effectivement la transformée de Fourier du facteur de forme d'un objet isolé. Ainsi, par exemple, l'intensité diffusée par une sphère isolée de rayon $R$ (en pratique, une solution très diluée et monodisperse de sphères en suspension dans un liquide) est proportionnelle au carré de la fonction de Bessel sphérique du premier ordre d'argument $Q R$.

Il est intéressant de noter que, pour ce cas extrême de simplicité (sphère isolée à l'origine), la fonction de corrélation de paires $g(r)$ se réduit à la fonction d'Heaviside égale à 1 pour $r>R$.

En conséquence, le facteur de structure, $S(Q)$, défini par:

$$
S Q=1+N \int[g(r)-1] \exp (i \vec{Q} \cdot \vec{r}) \mathrm{d} \vec{r}
$$

est aussi, au signe près, la même fonction de Bessel; c'est à dire, le facteur de forme, souvent noté $P(Q)$, est naturellement égal au carré de $S(Q)-1$. 
Dans le cas où la concentration de sphères en suspension est suffisamment grande pour que leur interaction génère une corrélation entre les positions de chaque sphère, la fonction de corrélation de paires des centres des sphères sera différente de 1, sauf pour $r$ suffisamment grand. On peut montrer que, dans ce cas, l'intensité diffusée peut se factoriser sous la forme :

$$
I Q=N K^{2} V^{2} P(Q) S(Q)
$$

où $K$ représente le "contraste", différence entre la densité de longueur de diffusion cohérente des sphères et celle du milieu continu, $V$ est le volume de chaque sphère et $N$ leur nombre par unité de volume. Dans cette expression, et suivant l'usage courant en DNPA, $P(Q)$, carré du facteur de forme, ainsi que $S(Q)$, n'ont pas d'unités et $I(Q)$ s'exprime comme une section efficace par unité de volume de l'échantillon, soit dans les unités de l'inverse d'une longueur. Cette convention est bien adaptée à l'étude de systèmes complexes.

L'expression précédente est souvent appliquée à des situations qui ne respectent pas les hypothèses nécessaires à sa dérivation, à savoir, des particules sphériques monodisperses. Il est pourtant possible d'établir une expression encore exacte si les particules diffusantes ont un centre de symétrie. On écrit alors :

$$
I Q=N K^{2} \overline{F^{2}(Q)}\left\{1+\frac{(\overline{F(Q)})^{2}}{\overline{F^{2}(Q)}}[S(Q)-1]\right\}
$$

où $F(Q)$ est le facteur de forme des particules, la barre au dessus des variables signifie la moyenne sur les orientations des particules par rapport à, $\vec{Q}$ et $P(Q)=\overline{F^{2}(Q)}$. Bien sûr, dans le cas de particules sphériques $(\overline{F(Q)})^{2}=\overline{F^{2}(Q)}$.

\section{FLUCTUATIONS DE DENSITÉ ET FLUCTUATIONS DE CONCENTRATION}

Un concept important en Physique Statistique est celui de fluctuations, qui sont l'équivalent de la variance dans un ensemble numérique. D'une manière très générale, toute expérience de DNPA mesure des "fluctuations" de la densité de la longueur de diffusion cohérente des atomes présents dans l'échantillon. Pourtant, le mot "fluctuations" est normalement utilisé dans une acception restreinte au voisinage de points critiques ou de démixtion.

La situation la plus simple est celle d'un fluide homogène. La densité (en nombre) de molécules, $N / V$, évalué sur un volume $V$ macroscopique s'écrit donc $\rho=N / V$. Pourtant, à cause du désordre inhérent, si le volume $V$ est petit, on observe des fluctuations du nombre $N$. Ces fluctuations en nombre correspondent à la compressibilité isotherme, $\chi_{T}$, et on déduit en Physique Statistique la relation :

$$
\frac{\left\langle(N-\langle N\rangle)^{2}\right\rangle}{\langle N\rangle}=\rho k_{B} T \chi_{T}
$$

où $k_{B}$ est la constante de Boltzmann et $T$ est la température. Avec la convention usuelle sur les "unités absolues" de l'intensité diffusée, le facteur de structure extrapolé à $Q=0$ est donné par :

$$
S(Q \rightarrow 0)=\left(\frac{N}{V}\right)^{2}\langle b\rangle^{2} k_{B} T \chi_{T}=\rho^{2}\langle b\rangle^{2} k_{B} T \chi_{T}
$$

où b2 est le carré de la moyenne des longueurs de diffusion cohérentes des atomes constitutifs des molécules. En pratique, il s'agit d'une intensité très faible. Ainsi pour le $\mathrm{D}_{2} \mathrm{O}, S(0)=0.0071 \mathrm{~cm}^{-1}$.

Près d'un point critique, les fluctuations, et donc la compressibilité isotherme, sont très grandes et on peut leur associer une longueur de corrélation, $\xi$. Dans ces cas, l'expression précédente s'écrit :

$$
S(Q \rightarrow 0)=\frac{\rho^{2}\langle b\rangle^{2} k_{B} T \chi_{T}}{1+Q^{2} \xi^{2}}
$$


On peut interpréter $\xi$ comme la taille caractéristique du volume minimum au-delà duquel le liquide est homogène.

Ces considérations s'appliquent mutatis mutandis aux mélanges de liquides auxquels l'expression précédente s'applique si l'on remplace la longueur de diffusion moléculaire par le contraste entre les molécules des deux composants et la compressibilité isotherme par une compressibilité "osmotique". Les valeurs observables en pratique sont souvent très grandes si on les compare aux fluctuations de densité des liquides simples, quoique dépendants du contraste.

Dans un système complexe, la première opération effectuée sur les données consiste à soustraire la diffusion incohérente et la contribution des fluctuations de densité à l'intensité diffusée ce qui se fait couramment par une mesure indépendante de l'intensité diffusée par le seul milieu continu. Ceci suppose implicitement un découplage total entre les fluctuations de densité et les structures à étudier.

Dans l'expression précédente, le facteur $1 /\left(1+Q^{2} \xi^{2}\right)$ est une Lorentzienne, transformée de Fourier de l'exponentielle exp $(-r / \xi)$, qui est la forme la plus simple que l'on puisse adopter pour la décroissance d'une fonction de corrélation.

Ce comportement est souvent source de confusion ou d'erreurs car, pour $Q \xi<1$, il ne se distingue pas facilement de la loi de Guinier qui s'applique à la diffusion de particules monodisperses de rayon de giration $R_{g}$. En effet, un simple développement, valable pour des valeurs de $Q$ petits, donne $R_{g}^{2}=3 \xi^{2}$. Ceci n'est pas surprenant parce que l'intensité diffusée, $I(Q)$, est une fonction paire de $Q$, avec le développement $I(Q)=I(0)\left[1 \pm a Q^{2}+\ldots\right]$ valable aux petites valeurs de $Q$. L'interprétation du coefficient $a$ ne peut se faire que dans le cadre d'un modèle physique ou, mieux, avec l'analyse de données s'étendant sur des gammes de $Q$ suffisamment larges.

En tout cas, les fluctuations sont toujours présentes dans tout système relevant de la Physique Statistique, dont les liquides, les solutions, les gels, les verres, les solutions colloïdales, les polymères et toute la "matière molle". Si leur longueur de corrélation est suffisamment grande, elles se caractérisent par une diffusion centrale de forme Lorentzienne. En pratique, la représentation de $[I(Q)]^{-1}$ en fonction de $Q^{2}$ est une droite dont la pente fournit la longueur de corrélation $\xi$, sans que la normalisation en unités absolues soit nécessaire.

On peut remarquer que la détermination de $\xi$ se fait tout naturellement dans l'espace réciproque. En effet, la composante exponentielle présente dans le $g(r)$, transformée de Fourier de la Lorentzienne centrée à $Q=0$ est à peine décelable. Cela explique aussi la difficulté souvent constatée à étudier en détail les fluctuations par des méthodes de simulation dans l'espace réel.

On ne saurait trop insister sur le fait qu'une longueur de corrélation de fluctuations n'est pas du tout assimilable à une quelconque longueur apparente dans l'espace réel. Aussi, la valeur de $S(0)$ est, comme nous l'avons vu, associée à une fonction réponse, la compressibilité.

Les fluctuations les plus élémentaires, car présentes même dans les liquides homogènes, sont les fluctuations en nombre, associées à la compressibilité isotherme. Dans le cas des fluctuations de concentration, deux ou plus espèces chimiques sont présentes et c'est leur contraste, en termes de longueur de diffusion cohérente, qui contribue à la diffusion aux petits angles. On peut donc imaginer une interface de topologie forcément complexe mais univoquement définie séparant les espèces les unes des autres. Mais les dimensions caractéristiques de cette interface ne dépassent pas la taille moléculaire et ne contribuent à aucune diffusion du type Porod. La diffusion garde donc la forme de la Lorentzienne sur de très larges domaines de $Q$.

Pourtant, la substitution isotopique, dans la mesure où elle n'altère pas les propriétés du système, peut fournir une méthode pour séparer les fluctuations de densité des fluctuations de concentration. En effet, si l'on annule le contraste entre deux composants d'un mélange, on élimine la contribution des fluctuations de concentration à l'intensité diffusée. Cette méthode revient à utiliser le formalisme de Bhatia et Thornton, qui consiste à écrire l'intensité diffusée en fonction des fluctuations en nombre $(\mathrm{NN})$ et en concentration $(\mathrm{CC})$ :

$$
S(Q)=S_{N N}(Q)+S_{C C}(Q)
$$


Dans le cas où l'on efface le contraste en les deux constituants $(\Delta b=0), S(Q)=S_{N N}(Q)$, tandis que, si l'on prépare un échantillon de densité de longueur de diffusion moyenne nulle $(\langle b\rangle=0), S(Q)=$ $S_{C C}(Q)$.

Pour exemplifier les possibilités de la diffusion neutronique dans un cas académique, nous montrons ci-dessous le cas d'un système contenant deux types de particules (deux valeurs de $b$ ou de densité de $b$ différentes). On y décèle, a priori, des fluctuations en nombre et des fluctuations des concentrations locales des deux espèces. Ainsi, dans le premier cas, les "fluctuations de densité" font apparaître à chaque instant des distributions spatiales des espèces non homogènes. Ceci est d'ailleurs à l'origine d'une compressibilité isotherme. Par ailleurs, les potentiels entre les deux types de particules étant différents, on aura aussi des régions de ségrégation, soit des "fluctuations de concentration" qui expliquent la compressibilité osmotique. Il est clair qu'un marquage isotopique convenable peut éliminer la différence de section efficace de diffusion cohérente entre les deux types de particules (via des mélanges isotopiques convenables). Alors, l'intensité diffusée ne mesurera que les fluctuations de densité.

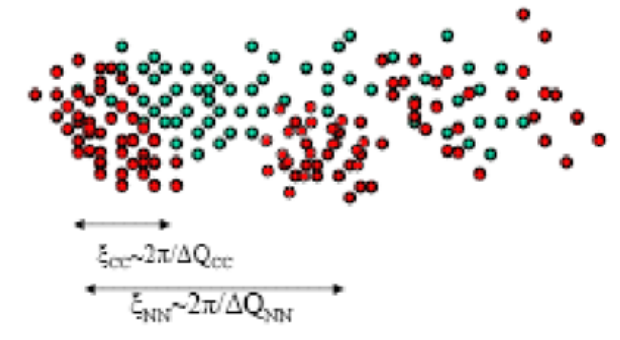

$$
\begin{aligned}
& S(Q)=S_{N N}(Q)+S_{C C}(Q) \\
& \text { Pour } \Delta b=0(\bullet=\bullet), S(Q)=S_{N N}(Q) \\
& \lim _{\mathrm{Q} \rightarrow \infty} S_{N N}(Q)=1 \\
& \lim _{\mathrm{Q} \rightarrow \infty} S_{C C}(Q)=c_{A} c_{B} \\
& S_{N N}(0) \alpha\left\langle\Delta \mathrm{N}^{2}\right\rangle \\
& \quad S_{C C}(0) \alpha\left\langle\Delta c^{2}\right\rangle
\end{aligned}
$$

Bien sûr, ce type d'approche est mis à profit dans des situations très diverses, telles que la détermination de facteurs de structure partiels, de parties de macromolécules, de conformation de polymères, etc.

Pour terminer, notons que les deux types de fluctuations et leur importance ne sont pas forcément "intuitives"! A titre d'exemple, et comme cela a été évoqué ci-dessus, un mélange de polystyrène hydrogéné et deutéré se sépare lentement au cours du temps et présente donc des fluctuations de concentration. Au contraire, la diffusion d'un mélange de $\mathrm{H}_{2} \mathrm{O}$ et $\mathrm{D}_{2} \mathrm{O}$, qui est en fait, un mélange de trois molécules, $\mathrm{H}_{2} \mathrm{O}, \mathrm{D}_{2} \mathrm{O}$ et $\mathrm{HDO}$ est uniquement due aux fluctuations de densité. Ce dernier exemple est assez pédagogique puisqu'il montre que la seule présence de "clusters" instantanés d'une espèce ne suffit pas à l'existence d'une diffusion spécifique. Encore une fois, notons qu'il faut en plus une cohérence, et qu'elle n'est bien apparente que dans l'espace réciproque. 